\section{Identification of lung sliding: a basic ultrasound technique with a steep learning curve}

\author{
MATEJ STRNAD • SABINA ZADEL • ZALIKA KLEMENC-KETIS • \\ GREGOR PROSEN
}

\begin{abstract}
MATEJ STRNAD $(\square)$ SABINA ZADEL・ GREGOR PROSEN Prehospital Unit, Center

for Emergency Medicine

Phone: (+386) 51225448

Fax: (+386) 22286585
\end{abstract} Ulica talcev 9, 2000 Maribor, Slovenia

E-mail: strnad.matej78@gmail.com

MATEJ STRNAD • ZALIKA KLEMENC-KETIS •

GREGOR PROSEN

Medical Faculty

University of Maribor, Slovenia

SABINA ZADEL •

GREGOR PROSEN

Faculty of Health Sciences

University of Maribor, Slovenia

ZALIKA KLEMENC-KETIS

Department of Family Medicine Medical Faculty, University of

Ljubljana,Slovenia

\begin{abstract}
Introduction. One of the basic premises of sonographic lung imaging is the concept of lung sliding. Identification of clear lung sliding excludes pneumothorax (PTX) at that specific local point.

Methods. Fifty-seven 4th year medical students were given a 20-minute lecture on sonographic identification of lung sliding and exclusion of PTx. After the lecture, students were asked to correctly position the probe, identify shown structures and on each attempt (six attempts in a row) state whether lung sliding is present or not.

Results. There were 57 students in the sample. Fifty students (87.7\%) successfully positioned the probe (all 4 positions) for PTx identification. All but five students (91.2\%) recognized the anatomic structures of the thorax. Mean number of correctly identified cases per student was $5.1 \pm 1.1$. In 292 (85.4\%) cases, the answer was correct. In 298 (87.1\%) cases, students were confident in the correct answer. Students who were confident in the right answer gave the right answer significantly more often when compared to others $(90.3 \%$ vs. $52.3 \%, p<0.001)$. Sensitivity of this method for 4 th year medical students was $82.6 \%$ and its specificity was $87.9 \%$. For correct identification of lung sliding in the sixth attempt, students on average needed 4.5 correct attempts.

Conclusion. Our study suggests that 4th year medical students with no prior experience in lung ultrasonography can easily acquire knowledge and skills needed to detect thoracic wall structures and identify lung sliding with a high degree of sensitivity and specificity.
\end{abstract}

Key words: medical students, ultrasonography, pneumothorax

\section{Introduction}

Point-of-care ultrasound is a recent concept where small portable ultrasound (US) machines are used to answer specific, focused clinical questions at the patient's bedside. Such focused concept of US examination can be performed by non-radiologist physicians and serves as an extension of physical examination and clinical acumen.

The concept of lung-sliding was first introduced by Lichtenstein in the 1980 's. The lung has been neglected for decades by sonographers because of ultrasonographic impenetrance through aerated lung tissue. During many years of research, Lichtenstein et al. have proven that lung can be 
imaged and its images interpreted - but the images mostly consist of predictable artifacts. Lichtenstein has shown that a healthy lung and various lung pathologies each have their specific sonographic pattern of either "true « images or sonographic artifacts. (1)

One of the basic premises of sonographic lung imaging is the concept of lung sliding. It represents a layer of visceral pleura sliding on parietal pleura lubricated by a small amount of pleural fluid (total $<50 \mathrm{ml}$ in healthy subjects). During breathing (spontaneous or mechanic), lung expansion creates a sliding movement between layers of pleura. US cannot identify a specific pleural layer. Lung sliding can be seen all over the lung surface where visceral pleura is directly adherent to the chest wall. (2)

Clear identification of lung sliding excludes pneumothorax at that specific local point. (3)

In our study, we tested how fast students learn to detect lung sliding. The study is based on the following statement from the International evidence-based recommendations of point-of-care lung ultrasound: "Ultrasound scanning for pneumothorax may be a basic ultrasound technique with a steep learning curve (level C)". (4)

\section{Materials and methods}

Statement of human rights

To an inquiry sent by the authors, the National Medical Ethics Committee of the Republic of Slovenia replied that the present study did not require ethics approval. Informed, written consent from each participant (medical student taking part in the study) was obtained.

Study design

This small pilot study was conducted as part of an elective emergency medicine module, dedicated to emergency ultrasound. For the majority of the students, this four-hour module was the first systematic exposure to US and certainly the first exposure to lung ultrasound (LUS). Fourth year pre-graduate students received a 20-minute lecture on the basics of LUS and the identification of lung sliding given by a certified

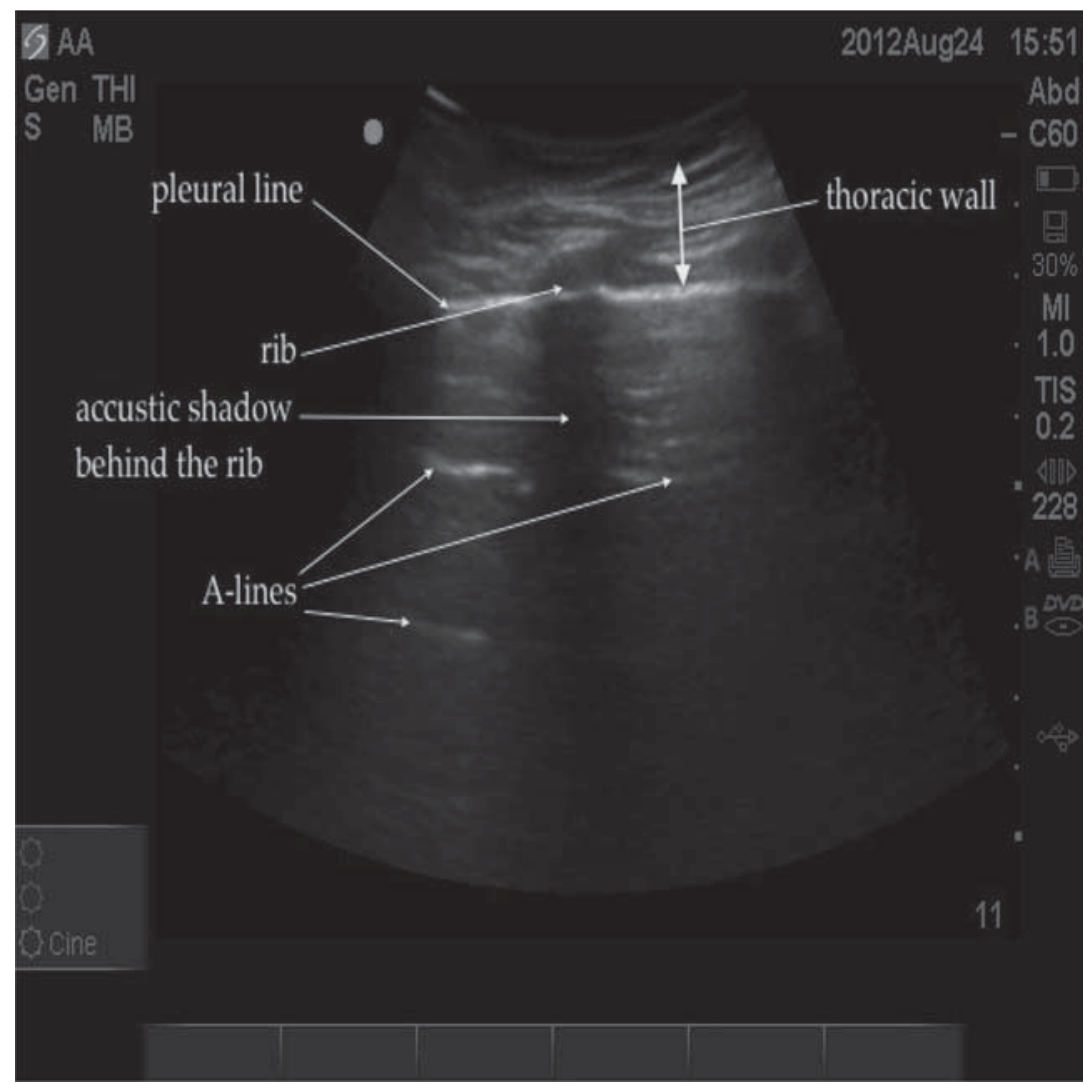

Figure 1. Anatomic structures of the thorax, which had to be recognized by the students after the lecture.

WINFOCUS (World Interactive Network Focused On Critical Ultrasound) instructor and lung US provider. This lecture contained basic US semiotics, probe position, image acquisition, structure identification and lung sliding identification. Tips and pitfalls were also given and students could ask questions at the end of the lecture.

Immediately after the lecture, the students individually went into an isolated room to assess the presence of lung sliding in a healthy volunteer. Only the chest of a female volunteer was exposed. One by one, students entered the room and were asked to position the probe as appropriate for pneumothorax identification (4 positions on anterior chest, bilaterally), as previously presented in the lecture. Students were then asked to recognize the anatomic structures of the thorax (figure 1). Subsequently, one of the authors positioned the probe in the right fifth intercostal space in the midaxillary line of the volunteer in a supine position. Thoracic US was performed using a microconvex C60x 2-5 $\mathrm{MHz}$ probe (Sonosite M Turbo ultrasound machine, Bothell, WA, USA). In the supine position, the authors could not find any artifacts which could help identify lung sliding (B lines, ring of pearls, lung pulse). Students were required to look at the screen perpendicular to the model (away from the model), so they could not take any clues from visual assessment of chest movements. The volunteer was asked to hold her breath or breathe spontaneously according to a computer generated random list. Every student had six attempts at lung sliding identification, with the probe in the same position. After each attempt, the student was asked to decide if lung sliding was present. The student replied with "lung sliding is present" or "lung sliding is not present". Maximum time to provide an answer in each attempt 


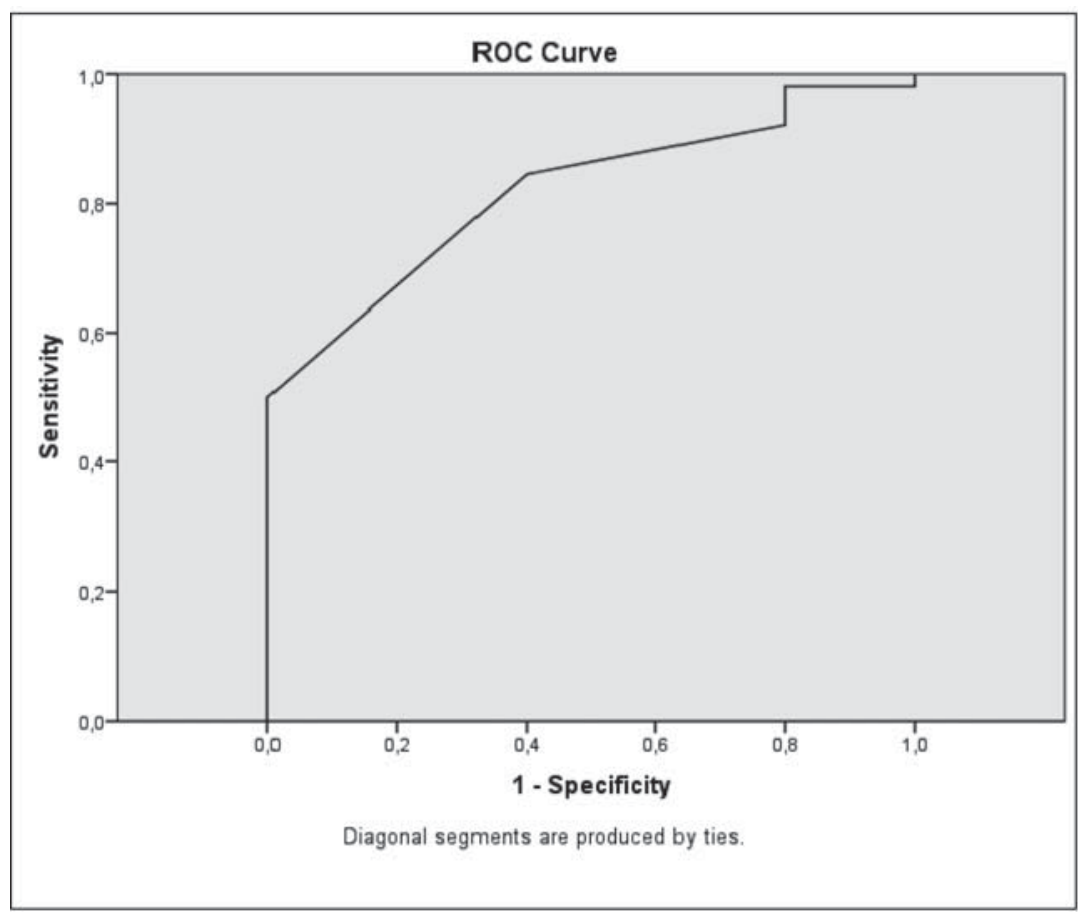

Legend: area under curve $=0.819 \pm 0.074, p=0.019$; coordinates of the curve: $\geq 0$ - sensitivity 0 , specificity $1.000, \geq 1.5$ - sensitivity 0.981 , specificity $0, \geq 2.5$ - sensitivity 0.981 , specificity $0.200, \geq 3.5$ - sensitivity 0.923 , specificity $0.200, \geq 4.5$ - sensitivity 0.846 , specificity $0.600, \geq 5.5$ - sensitivity 0.500 , specificity $1.000, \geq 7.0$ - sensitivity 0 , specificity 1.000

Figure 2. ROC curve for correct identification of lung sliding in sixth attempt.

was limited to 10 seconds. The validity of a student's answer was compared to the computer generated random list instructing the volunteer when to hold her breath or breathe spontaneously. We also measured the time needed to reach each decision and asked the students how confident they were about their decision ("confident" or "not confident").

SPSS 19.0 package (SPSS Inc, Chicago, IL) was used for the statistical analysis.

We used independent t-test, $x^{2}$-test and the receiver operating characteristic (ROC) curve analysis. In the latter, we regarded the sixth attempt to correctly diagnose lung sliding (yes/no) as the dependent variable. As the independent variables, we used the number of correctly diagnosed cases by each individual student. We also calculated the sensibility and specificity of the test. We regarded $p<0.05$ as statistically significant.

\section{Results}

There were 38 (66.7\%) females in the sample. Fifty students (87.7\%) successfully positioned the probe (all 4 positions) for pneumothorax identification. Fifty-two students (91.2\%) correctly recognized the anatomic structures of the thorax.

The mean number of correctly identified cases of lung sliding per student was $5.1 \pm 1.1$. The mean time it took to answer all cases was $3.1 \pm$ 1.9 seconds. The mean time it took to correctly answer all cases was 2.9 \pm 1.8 seconds. The mean time it took to incorrectly answer cases was 4.1 \pm 2.2 seconds. The difference was statistically significant $(t=3.503, p<$ 0.001). In 298 (87.1\%) cases, students were confident in the correct answer. In 292 (85.4\%) cases, the answer was correct (table 1). Students who were confident in their answer gave the right answer significantly more often when compared to others $(90.3 \%$ vs. $52.3 \%$, $\left.x^{2}=44.324, p<0.001\right)$. Sensitivity of this method (check the section Study design for details) for $4^{\text {th }}$ year medical students was $82.6 \%$ and its specificity was $87.9 \%$ (table 2).

For correct identification of lung sliding in the sixth attempt, students on average needed 4.5 correct attempts (figure 2).

\section{Discussion}

Our study was focused on recognition of structures of thoracic wall and lung sliding. We found that the above described method of introducing lung ultrasound to $4^{\text {th }}$ year medical students without previous experience in lung ultrasonography, is suitable and easy to comprehend, with high sensitivity and specificity. Medical students were confident about identifying lung sliding after a few attempts. More precisely, our data show that after 4.5 right answers, the student is skilled enough to identify lung sliding. These findings are similar to Lyon et al., who showed that pre-hospital critical care providers can quickly acquire and retain skills necessary to detect lung sliding by the use of ultrasound. (5)

Pneumothorax (PTx) is a potentially deadly disease ranging from small PTx that might not require any treatment, to rapidly fatal obstructive shock of tension PTx. Diagnosis of PTx is clinical and based on auscultation and percussion, especially of tension PTx. (6) Specificity and sensitivity of chest $X$ ray imaging of PTx is poor, shown by many studies. $(7,8)$

Ultrasound signs of PTx are primarily based on the fact that free air will accumulate in the nondependent areas of the thoracic cavity. In a supine patient, a PTx will be found anteriorly at the highest point of the thoracic cavity. (9) The other principle is that during normal spontaneous breathing or mechanical ventilation the visceral pleura move against the parietal pleura. This movement can be detected by ultrasound and is defined as lung sliding. The presence of lung sliding excludes a PTx. (3)

According to Lichtenstein et al. (the BLUE Protocol), in a patient with acute 
Table 1. Characteristics of students' attempts to correctly diagnose lung sliding.

\begin{tabular}{llll}
\hline Attempt No. & $\begin{array}{l}\text { Mean (standard deviation) of time (sec) } \\
\text { needed for answer }\end{array}$ & $\begin{array}{l}\text { No (\%) of correctly recognized } \\
\text { cases }\end{array}$ & $\begin{array}{l}\text { Confidence in correct } \\
\text { answer }\end{array}$ \\
\hline 1 & $2.7(1.9)$ & $45(78.9)$ & $42(73.7)$ \\
2 & $3.0(1.8)$ & $50(87.7)$ & $53(93.0)$ \\
3 & $3.4(1.9)$ & $45(78.9)$ & $47(82.5)$ \\
4 & $2.9(1.8)$ & $49(86.0)$ & $53(93.0)$ \\
5 & $3.3(2.0)$ & $51(89.5)$ & $50(87.7)$ \\
6 & $3.3(1.8)$ & $52(91.2)$ & $53(93.0)$ \\
\hline
\end{tabular}

Table 2. Specificity and sensibility of this diagnostic method for 4th year medical students.

\begin{tabular}{lll}
\hline & $\begin{array}{l}\mathrm{N}(\%) \text { of students who gave a positive } \\
\text { answer }\end{array}$ & $\begin{array}{l}\mathrm{N}(\%) \text { of students who gave a negative } \\
\text { answer }\end{array}$ \\
\hline Lung sliding present & $138(40.4)$ & $29(8.5)$ \\
Lung sliding absent & $29(8.5)$ & $152(44.4)$ \\
\hline
\end{tabular}

respiratory failure anterior lung sliding is checked first. (10) Its presence excludes PTx. On the other hand, absent lung sliding associated with $A$ lines (horizontal repetition of the pleura line recurring at regular intervals - reverberation artifacts from pleura) suggests PTx and in that case immediate exclusion of PTx is mandatory.

Lung sliding can be also shown by using M-mode Doppler. A normal image is called the "seashore sign", with the thoracic wall, represented as horizontal lines, and the adherent, ventilated lung generating a sandy pattern on M-mode. When a PTx is present, the M-mode Doppler will show only repeating horizontal linear lines, identified as "stratosphere sign" by Lichtenstein. (10)

Although the presence of lung sliding is sufficient to rule out PTx, the absence of lung sliding may be seen in other conditions such as apnea, a main stem intubation, severe bullous chronic obstructive pulmonary disease, pleurodesis, pulmonary fibrosis, consolidated pneumonia, atelectasis, acute respiratory distress syndrome (ARDS), etc. Thus the absence of lung sliding, especially defined in only one intercostal space, is not by itself diagnostic of a PTx. The clinician should examine several more intercostal spaces, moving the trans- ducer more inferiorly and lateral searching for the lung point, $(6,11)$ which can be identified by the presence of lung sliding on one side and the lack of lung sliding on the other. (12)

Another sonographic finding, called B lines, rules out a PTx as well. B-lines are defined as discrete laser-like vertical hyperechoic reverberation artifacts that arise from the pleural line, extend to the bottom of the screen without fading, and move synchronously with lung sliding. Presence of B lines alone has a sensitivity and negative predictive value for PTX up to $100 \%$. (13)

The lung pulse is a vibration or slight movement visible at the pleural line, in rhythm with the heart beat, visible when lung sliding is absent. Lung pulse is an indirect proof of inflated but nonventilated lung, adherent to parietal pleura, thus excluding PTx. Lung pulse can therefore be a valuable sign in ruling out a PTx. (14)

Lung ultrasound allows accurate diagnosis of many pulmonary pathologies in critically ill patients such as interstitial syndrome, (15) alveolar interstitial syndrome, (16) pleural effusion, alveolar consolidation, $(2,17)$ pulmonary embolism, (18) etc.

It could also be used for monitoring the success of treatment in patients with interstitial syndrome (19) or in patients treated with positive end expiratory pressure (PEEP), showing reaeration of the lung. (20) In addition, the evaluation of lung sliding can help identify proper tracheal tube placement. The technique relies on seeing lung sliding on both sites of the thoraces while ventilating. $(21,22)$

Our study has several limitations. Firstly, the study group was small. The study group was selected only from $4^{\text {th }}$ year medical students. The results would probably be better with $5^{\text {th }}$ or $6^{\text {th }}$ year medical students or attending doctors, who have more clinical experience and confidence. Secondly, the number of attempts was small. With more attempts the students would be more confident and the percentage of right answers would probably be higher. Thirdly, students did not place the probe on the volunteer by themselves. It was already placed on the thorax by one of the authors.

\section{Conclusion}

Our study suggests that $4^{\text {th }}$ year medical students with no prior experience in lung ultrasonography can acquire skills to detect thoracic wall structures and identify lung sliding with a high degree of sensitivity and specificity. 


\section{ACKNOWLEDGMENTS}

We would like to thank Professor Peter Kokol (dean of the Faculty of Health Sciences at the University of Maribor) for providing the ultrasound machine (Sonosite M turbo equipped with appropriate probes) needed for the study.

\section{REFERENCES}

1. Lichtenstein D. Ultrasound in the management of thoracic disease. Crit Care Med 2007;35:S250-61.

2. Sperandeo M, Filabozzi P, Varriale A, Carnevale V, Piattelli ML, Sperandeo G, et al. Role of thoracic ultrasound in the assessment of pleural and pulmonary diseases. J Ultrasound 2008;11:39-46.

3. Lichtenstein D, Menu Y. A bedside ultrasound sign ruling out pneumothorax in the critically ill: Lung sliding. Chest 1995;108:1345-8.

4. Volpicelli G, Elbarbary M, Blaivas M, Lichtenstein D, Mathis G, Kirkpatrick AW, et al. International evidence-based recommendations for point-of-care lung ultrasound. Intensive Care Med 2012;38:577-91.

5. Lyon M, Walton P, Bhalla V, Shiver SA. Ultrasound detection of the sliding lung sign by prehospital critical care providers. Am J Emerg Med 2012;30:485-8

6. Perera P, Mailhot T, Riley D, Mandavia D. The RUSH Exam: Rapid Ultrasound in Shock in the Evaluation of the Critically ill. Emerg Med Clin North Am 2010;28:39-56

7. Rowan KR, Kirkpatrick AW, Liu D, Forkheim KE, Mayo JR, Nicolaou S. Traumatic pneumothorax detection with thoracic US: correlation with chest radiography and CT - initial experience. Radiology 2002;225:210-4.

8. Soldati G, Testa A, Sher S, Pignataro G, La Sala M, Gentiloni Silveri N. Occult Traumatic Pneumothorax: Diagnostic Accuracy of Lung Ultrasonography in the Emergency Department. Chest 2008;133:204-11.

9. Mennicke M, Gulati K, Oliva I, Goldflam K, Skali H, Ledbetter S, et al. Anatomical distribution of traumatic pneumothoraces on chest computed tomography: implications for ultrasound screening in the ED. Am J Emerg Med 2011;30:1025-31.

10. Lichtenstein D, Mezičre GA. Relevance of lung ultrasound in the diagnosis of acute respiratory failure: the BLUE protocol. Chest 2008; $134: 117-25$.

11. Lichtenstein D, Meziere G, Biderman P, Gepner A. The žžlung point": an ultrasound sign specific to pneumothorax. Intensive Care Med 2000;26:1434-40

12. Zhang M, Liu ZH, Yang JX, Gan JX, Xu SW, You XD, et al. Rapid detection of pneumothorax by ultrasonography in patients with multiple trauma. Crit Care 2006;10:R112.

13. Lichtenstein D, Meziere G, Biderman P, Gepner A. The comet-tail artifact: an ultrasound sign ruling out pneumothorax. Intensive Care Med 1999;25:383-8

14. Lichtenstein D, Lascols N, Prin S, Mezičre G. The "lung pulse": an early ultrasound sign of complete atelectasis. Intensive Care Med 2003;29:2187-92.

15. Agricola E, Bove T, Oppizzi M, Marino G, Zangrillo A, Margonato A, et al. "Ultrasound comet tail images": A marker Of Pulmonary Edema: A Comparative Study With Wedge Pressure And Extravascular Lung Water. Chest 2005;127;1690-5.

16. Lichtenstein D, Mezičre G, Biderman P, Gepner A, Barre O. The Comet-tail Artifact. An Ultrasound Sign of Alveolar-Interstitial Syndrome. Am J Respir Crit Care Med 1997;156:1640-6.

17. Parlamento S, Copetti R, Di Bartolomeo S. Evaluation of lung ultrasound for the diagnosis of pneumonia in the ED. Am J Emerg Med 2009;27:379-84.

18. Reissig A, Heynes JP, Kroegel C. Sonography of lung and pleura in pulmonary embolism: sonomorphologic characterization and comparison with spiral CT scanning. Chest 2001;120:1977-83.

19. Volpicelli G, Caramello V, Cardinale L, Mussa A, Bar F, Frascisco MF. Bedside ultrasound of the lung for the monitoring of acute decompensated heart failure. Am J Emerg Med 2008;26:585-91.

20. Tsubo T, Sakai I, Suzuki A, Okawa H, Ishihara H, Matsuki A. Density detection in dependent left lung region using transesophageal echocardiography. Anesthesiology 2001;94:793-8.

21. Sim SS, Wan-Ching L, Chou HC, Chong KM, Liu SH, Wang CH, et al. Ultrasonographic lung sliding sign in confirming proper endotracheal intubation during emergency Intubation. Resuscitation 2012;83:307-12.

22. Breitkreutz R, Seibel A, Zechner PM. Ultrasound-guided evaluation of lung sliding for widespread use? Resuscitation 2012;83:273-4. 